\title{
Pharmacokinetics of dolutegravir in HIV-seronegative subjects with severe renal impairment
}

\author{
Stephen Weller • Julie Borland • Shuguang Chen • Mark Johnson • \\ Paul Savina • Brian Wynne $\cdot$ Toshihiro Wajima • \\ Amanda F. Peppercorn • Stephen C. Piscitelli
}

Received: 22 July 2013 / Accepted: 16 September 2013 /Published online: 6 October 2013

(C) The Author(s) 2013. This article is published with open access at Springerlink.com

\begin{abstract}
Purpose Dolutegravir (DTG), an unboosted HIV integrase inhibitor (INI), is metabolized by UGT1A1 and to a minor extent by CYP3A. Renal elimination of unchanged DTG is very low $(<1 \%)$. As renal impairment may affect pharmacokinetics (PK), even for drugs primarily metabolized or secreted in bile, this study investigated the effect of renal impairment on the PK of DTG.

Methods This was an open-label, single-dose study of oral DTG $50 \mathrm{mg}$ administered to subjects with severe renal impairment (creatinine clearance [CLcr] $<30 \mathrm{~mL} / \mathrm{min}$; not on dialysis) and to healthy controls (CLcr $>90 \mathrm{~mL} / \mathrm{min}$ ) matched for gender, age and body mass index ( 8 subjects per group). Serial PK samples were collected up to $72 \mathrm{~h}$ post-dose for determination of DTG and DTG-glucuronide (DTG-Gluc) concentrations in plasma. DTG unbound fraction in plasma was determined at 3 and $24 \mathrm{~h}$. PK parameters were determined by non-compartmental methods and compared between groups by analysis of covariance.
\end{abstract}

ClinicalTrials.gov identifier: NCT01353716

These data were presented in part at the 53rd Interscience Conference on Antimicrobial Agents and Chemotherapy, September 10-13, 2013,

Denver, CO, USA

Electronic supplementary material The online version of this article (doi:10.1007/s00228-013-1590-9) contains supplementary material, which is available to authorized users.

S. Weller $\cdot$ J. Borland $\cdot$ S. Chen $\cdot$ M. Johnson $\cdot$ P. Savina $\cdot$

A. F. Peppercorn · S. C. Piscitelli $(\square)$

GlaxoSmithKline, Research Triangle Park, GlaxoSmithKline,

5 Moore Drive, Research Triangle Park, NC 27709, USA

e-mail: stephen.c.piscitelli@gsk.com

B. Wynne

GlaxoSmithKline, Upper Providence, Collegeville, PA, USA

T. Wajima

Shionogi \& Co., LTD, Osaka, Japan
Results DTG was well tolerated with a low incidence of Grade 1 adverse events. DTG PK parameters showed significant overlap between groups. DTG mean exposure was lower in subjects with severe renal impairment compared to healthy, matched subjects: $\mathrm{AUC}(0-\infty)$ and $\mathrm{Cmax}$ were $40 \%$ and $23 \%$ lower, while mean DTG-Gluc was increased. Renal impairment did not affect DTG fraction unbound in plasma.

Conclusions The modest reductions in mean PK exposures for DTG and increases for DTG-Gluc in the severe renal impairment group are not considered clinically significant. DTG does not require dose adjustment in patients with renal impairment.

Keywords Dolutegravir · Pharmacokinetics · Renal impairment $\cdot$ Protein binding

\section{Introduction}

Dolutegravir (DTG) is an investigational integrase inhibitor (INI) for treatment of HIV infection which has demonstrated safety and efficacy in Phase 3 trials [1-3]. Its unique resistance profile supports its antiviral activity in patients who have previously failed treatment with the INI raltegravir [4].

The pharmacokinetic (PK) profile of DTG is characterized by a long half-life supporting once-daily dosing in INI-naïve subjects, no requirement for boosting with ritonavir, and a well-characterized $\mathrm{PK} /$ pharmacodynamic relationship demonstrated in short-term monotherapy [5, 6]. DTG is primarily metabolized via glucuronidation by UGT1A1 in the liver, and to a minor extent by CYP3A. It is greater than $99 \%$ bound to plasma proteins [7]. DTG is the predominant circulating compound in plasma ( $>97 \%$ of drug-related components) and renal elimination of unchanged drug is extremely low $(<1 \%$ of the dose) [8]. Approximately $53 \%$ of a dose is recovered as DTG in feces and approximately $31 \%$ is excreted in the urine 
primarily as DTG-glucuronide (DTG-Gluc) and other minor metabolites [8].

Severe renal impairment can impact the PK of drugs which are primarily metabolized or secreted in bile [9]. Current regulatory guidance recommends evaluation of drug PK in subjects with renal impairment, even for drugs that are primarily metabolized [10]. Accordingly, this study investigated if renal impairment significantly affects the PK of DTG.

\section{Methods}

Study design and participants

This was a single-center, Phase I, single-dose, open-label study in human subjects with severely impaired renal function who are not on renal replacement therapy, in comparison to a matched group of healthy subjects with normal renal function. Severe renal impairment was defined as a creatinine clearance (CLcr) $<30 \mathrm{~mL} / \mathrm{min}$ based on 24-hour urine creatinine clearance within 30 days of the treatment period. Healthy subjects were required to have CLcr $>90 \mathrm{~mL} / \mathrm{min}$ based on 24-hour urine creatinine clearance within 30 days of the treatment period and were matched to severe renal impaired subjects for gender, age ( \pm 5 years) and body mass index $( \pm 25 \%)$. As race/ethnicity has not been shown to affect DTG pharmacokinetics, this was not used as a factor for matching [11]. A sample size of eight subjects per group was chosen based on regulatory guidance for studies in renal impairment [10].

HIV-seronegative male and female participants between 18 to 70 years of age and with a body weight $\geq 50 \mathrm{~kg}$ and $\mathrm{BMI}$ in the range of 19 to $38 \mathrm{~kg} / \mathrm{m}^{2}$ were eligible for enrollment. Subjects with renal impairment were enrolled if they met the CLcr inclusion criteria and had laboratory test results that were considered clinically stable by the principal investigator (PI). Renal impairment subjects were excluded for recent hepatitis B infection, a positive HIV antibody and preexisting conditions (other than renal impairment) that might interfere with normal gastrointestinal anatomy or motility and/ or those that could interfere with absorption, metabolism, or excretion of the study drug. All subjects could not have consumed red wine, Seville oranges or grapefruit products within 7 days prior to the dose of study medication until the collection of the last PK sample, but renally impaired subjects were permitted to use concomitant medications that were considered medically necessary by the PI and did not have the potential to affect DTG exposure, as determined by effects on DTG metabolic pathways. Antacids, vitamins, and supplements were held on the day of dosing.

Healthy participants were determined to be eligible for inclusion based on physical exam, medical history, and laboratory evaluation. They could not receive any prescription or nonprescription drugs or consume red wine, Seville oranges or grapefruit products within 7 days prior to the dose of study medication until the collection of the last PK sample. Furthermore, exclusion criteria for healthy participants included a positive pre-study drug screen for drugs of abuse.

Subjects received a single $50 \mathrm{mg}$ tablet of DTG in the morning in a fasted state and were housed in the study center until collection of the last PK sample. Blood samples for determination of total DTG and DTG-Gluc plasma concentrations were collected pre-dose and at 1, 2, 3, 4, 5, 6, 8, 12, 24,48 , and $72 \mathrm{~h}$ post-dose. Additional plasma samples were collected at 3 and $24 \mathrm{~h}$ post-dose for measurement of unbound plasma concentrations of DTG. Safety was assessed throughout the study by clinical and laboratory evaluations and at follow-up 7 to 10 days after administration of the DTG dose.

Written informed consent was obtained from all participants prior to conduct of any study-specific procedures and the protocol was approved by the IRB (Western Institutional Review Boards, Olympia, WA).

Bioanalytical methods

DTG $(M W=419.38)$ and DTG-Gluc $(M W=594.51)$ concentrations in plasma were determined by validated, high performance liquid chromatographic/tandem mass spectrometric methods following extraction with acetonitrile. Determination of unbound DTG concentration was conducted by equilibrium dialysis. Details of the analytical method and assay performance are provided in the supplementary document.

\section{Pharmacokinetic analysis}

Analysis of the DTG and DTG-Gluc concentration-time data was performed by noncompartmental PK methods (WinNonlin ${ }^{\circledR}$ Professional Edition 5.2; Pharsight Corporation, Mountain View, CA). Plasma DTG PK parameters calculated included area under the plasma concentration-time curve from time zero to infinity $(\mathrm{AUC}(0-\infty))$, area under the plasma concentration-time curve from time zero to the last quantifiable time point [AUC(0-t)], maximum observed plasma concentration (Cmax), time to $\mathrm{Cmax}$ (tmax), concentration at $24 \mathrm{~h}$ after dosing (C24), apparent oral clearance (CL/F), apparent volume of distribution $(\mathrm{Vz} / \mathrm{F})$, and terminal elimination phase half-life (t1/2). The same PK parameters were determined for DTG-Gluc except for CL/F, Vz/F and C24. Additionally, molar ratios of DTG-Gluc/DTG were determined for AUC(0-t) and Cmax, as was the DTG-Gluc/DTG t1/2 ratio.

Unbound fraction (fu) was calculated using the total and unbound plasma concentration of DTG data generated at 3 and $24 \mathrm{~h}$ post-dose for both healthy and renal impairment subjects using the following formula: $\mathrm{fu}=\mathrm{C}$ (unbound) $/ \mathrm{C}$ (total), where $\mathrm{C}$ (unbound) and $\mathrm{C}$ (total) are the unbound and total concentration of DTG in plasma, respectively. 
Statistical analysis

Statistical analysis was performed on the log-transformed PK parameters, except tmax and tlag. Analysis of covariance (ANCOVA) was performed using the SAS system (Version 9.1; SAS Institute Inc, Cary, NC) with gender as a fixed effect and with age and BMI as continuous covariates. For each logtransformed PK parameter, the point estimate and its associated $90 \%$ confidence interval (CI) was constructed for the difference between subjects with renal impairment (test) and matched healthy controls (reference). The difference in PK parameter and its $90 \% \mathrm{CI}$ was then exponentiated to obtain the ratio of geometric least squares means and its $90 \% \mathrm{CI}$ on the original scale. For DTG-Gluc/DTG ratios, HodgesLehmann estimates of difference and $90 \%$ CI were determined. Pearson correlation coefficients between creatinine clearance and DTG parameters and creatinine clearance and DTG-Gluc PK parameters were provided.

\section{Results}

Study population

Sixteen subjects ( 8 renal impairment; 8 healthy) were enrolled and completed all study assessments. Summary demographics are provided in Table 1. Groups were similar in age, gender and BMI given the matching criteria. There were 5 AfricanAmericans and 3 Caucasians in the renal impairment group and 2 African-Americans and 6 Caucasians in the healthy subject group. Concomitant medications in the renal impairment group were most commonly administered for treatment of hypertension, diabetes, and lipid disorders.

Pharmacokinetics

Mean concentration-time profiles of DTG and DTG-Gluc in subjects with and without renal impairment are shown in
Fig. 1. Summary DTG pharmacokinetic parameter estimates are provided in Table 2. Subjects with severe renal impairment had an average $40 \%$ lower plasma DTG AUC $(0-\infty)$ and $23 \%$ lower Cmax than subjects with normal renal function. This was accompanied by a mean elimination half-life that was shorter by $18 \%$ in the subjects with renal impairment compared to controls (12.7 vs $15.4 \mathrm{~h}$, respectively). There was considerable overlap in the plasma DTG PK parameters and in the direction of the change between renally impaired and healthy subjects. When compared to their matched controls, 6/8 healthy subjects had a higher AUC while 2/8 renally impaired subjects had a higher AUC. Values for AUC $(0-\infty)$ ranged from 13.6 to $46.7 \mu \mathrm{g}^{*} \mathrm{~h} / \mathrm{ml}$ in renal impairment subjects and from 14.1 to $60.9 \mu \mathrm{g}^{*} \mathrm{~h} / \mathrm{ml}$ in matched healthy subjects. For Cmax, 4/8 healthy subjects had a higher value while $3 / 8$ renally-impaired subjects had a higher value and one matched pair demonstrated similar a Cmax (1.8 vs $1.9 \mu \mathrm{g} / \mathrm{mL})$. Cmax ranged from 0.79 to $2.1 \mu \mathrm{g} / \mathrm{ml}$ in renal impairment subjects and from 0.82 to $3.2 \mu \mathrm{g} / \mathrm{ml}$ in healthy subjects. Variability in DTG PK parameters was similar between the two groups (Table 2). No significant correlation was observed between creatinine clearance and DTG PK parameters $(p>0.1)$.

The unbound fraction (fu) was similar between the severe renal impairment and normal renal function groups, but the GLS mean unbound plasma DTG concentrations were $14 \%$ lower at the 3-hour time point and $49 \%$ lower at the 24-hour time point in the renal impaired subjects (Table 2). Variability in DTG unbound fraction was higher in subjects with severe renal impairment. There was no apparent correlation between DTG unbound fraction and DTG total concentration in plasma, suggesting that protein binding is independent of DTG concentration over the range of concentrations observed in this study.

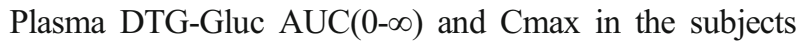
with severe renal impairment were approximately fourfold and threefold higher than those in the healthy subjects (Table 3). The median molar DTG-Gluc-to-DTG AUC ratio increased from 0.011 in healthy subjects to 0.053 in subjects with severe
Table 1 Subject demographics

$B M I$ body mass index, $S D$ standard deviation

\begin{tabular}{lll}
\hline Demographics & Severe Renal Impairment & Healthy Matched Controls \\
\hline $\begin{array}{l}\text { Age, mean (SD), y } \\
\text { Sex, } \mathrm{n}(\%)\end{array}$ & $56.8(7.42)$ & $56.1(8.32)$ \\
Female & $3(38)$ & $3(38)$ \\
Male & $5(63)$ & $5(63)$ \\
BMI, mean (SD), kg/m ${ }^{2}$ & $31.34(6.23)$ & $29.36(4.08)$ \\
Height, mean (SD), cm & $174.19(10.09)$ & $171.21(9.06)$ \\
Weight, mean (SD), kg & $94.48(17.09)$ & $86.59(17.05)$ \\
Race, $\mathrm{n}(\%)$ & & $2(25)$ \\
African American/African heritage & $5(63)$ & $6(75)$ \\
White, White/Caucasian/European heritage & $3(38)$ & \\
\hline
\end{tabular}




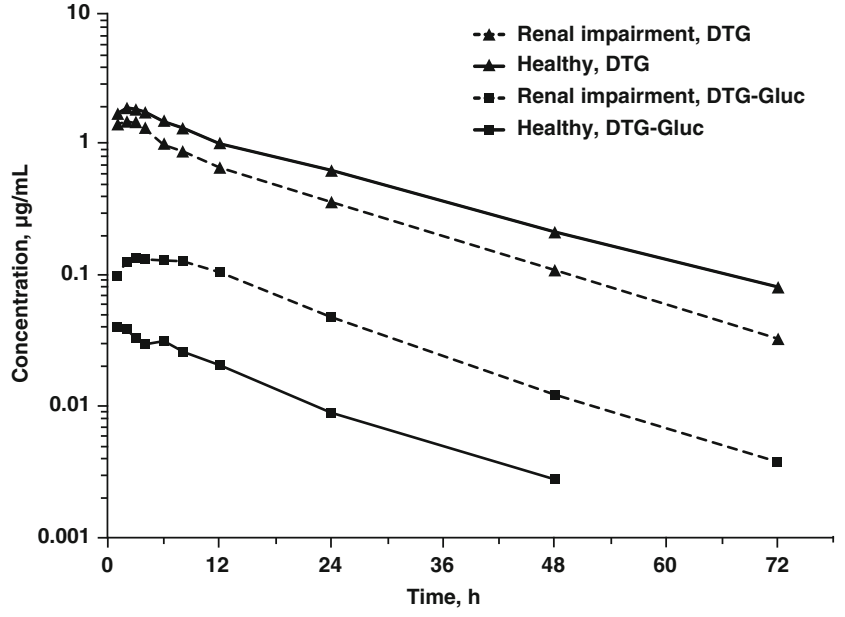

Fig. 1 Mean plasma DTG and DTG-Gluc concentration-time plots in subjects with and without renal impairment

renal impairment, and the median molar DTG-Gluc-to-DTG Cmax ratio increased from 0.015 in healthy subjects to 0.045 in subjects with severe renal impairment. The molar DTG-Glucto-DTG ratio of AUC and Cmax expressed as a percentage was $1-5.5 \%$, indicating that DTG is the predominant species in plasma, even in renal impairment. $\mathrm{AUC}(0-\mathrm{t})$ and $\mathrm{Cmax}$ ratios of DTG-Gluc to DTG were higher in subjects with severe renal impairment than healthy matched subjects. The $t 1 / 2$ ratio of DTG-Gluc to DTG is close to 1 in both groups of subjects, indicating that the terminal t1/2 of DTG-Gluc is similar to that of DTG, and that the formation-rate-limited kinetics for DTGGluc was not altered by severe renal impairment. There was a statistically significant negative correlation between creatinine clearance and DTG-Gluc PK parameters including AUC(0-t), $\operatorname{AUC}(0-\infty)$ and Cmax $(p<0.05$, correlation coefficients range -
0.54 to -0.60$)$, but not for the $\mathrm{t} 1 / 2$ of DTG-Gluc $(p>0.05$, correlation coefficient $=-0.07$ ).

\section{Safety}

DTG was well tolerated in both treatment groups. No deaths or serious adverse events occurred during this study. No subjects were withdrawn from the study due to adverse events (AEs) and all AEs were mild (Grade 1) in intensity. One subject with severe renal impairment experienced an $\mathrm{AE}$ (dizziness) considered by the investigator to be related to the study drug. The AE occurred approximately 15 min after dosing, resolved within $20 \mathrm{~min}$ and no action was taken. There were no drug-related AEs in the healthy subject group.

\section{Discussion}

DTG is primarily eliminated through metabolism, and renal elimination of unchanged drug represents less than $1 \%$ of the total dose administered. A study using a radio-labeled drug demonstrated that approximately $30 \%$ of the total oral dose is excreted in the urine, the majority of which is represented by a glucuronidated metabolite with a small percentage of oxidative and other minor metabolites [8]. Approximately $65 \%$ of the total oral dose is recovered in the feces, represented mainly by parent drug ( $53 \%$ of total dose) [8]. Given this metabolism and excretion profile, renal impairment would be expected to have a minor effect on DTG PK.

The results of this study showed that the mean total DTG plasma Cmax and AUC $(0-\infty)$ were $23-40 \%$ lower in subjects with severe renal impairment compared to those in matched,

Table 2 Comparison and summary of dolutegravir pharmacokinetic parameters and unbound concentrations

\begin{tabular}{llll}
\hline Parameter & $\begin{array}{l}\text { Renally impaired } \\
(\text { dolutegravir } 50 \mathrm{mg}, n=8)^{\mathrm{a}}\end{array}$ & $\begin{array}{l}\text { Healthy (dolutegravir } \\
50 \mathrm{mg}, n=8)^{\mathrm{a}}\end{array}$ & $\begin{array}{l}\text { Renally impaired vs. healthy, } \\
\text { GLS mean ratio }(90 \% \mathrm{CI})\end{array}$ \\
\hline $\mathrm{Cmax}, \mu \mathrm{g} / \mathrm{mL}$ & $1.50(34)$ & $1.86(45)$ & $0.774(0.532,1.13)$ \\
$\mathrm{AUC}(0-\infty), \mu \mathrm{g} \mathrm{h} / \mathrm{mL}$ & $23.5(48)$ & $37.1(58)$ & $0.601(0.370,0.975)$ \\
$\mathrm{CL} / \mathrm{F}, \mathrm{L} / \mathrm{hr}$ & $2.12(48)$ & $1.35(58)$ & $1.67(1.03,2.70)$ \\
$\mathrm{Vz} / \mathrm{F}, \mathrm{L}$ & $38.8(43)$ & $29.9(44)$ & $1.36(0.918,2.02)$ \\
$\mathrm{t} 1 / 2, \mathrm{~h}$ & $12.7(31)$ & $15.4(15)$ & $0.818(0.639,1.05)$ \\
tmax, h & $2.00(1.0-3.0)$ & $2.00(1.0-4.0)$ & $\mathrm{NA}$ \\
Unbound dolutegravir concentration at $3 \mathrm{~h}(\mu \mathrm{g} / \mathrm{mL})$ & $0.0122(21)$ & $0.0144(53)$ & $0.857(0.587,1.25)$ \\
Unbound dolutegravir concentration at $24 \mathrm{~h}(\mu \mathrm{g} / \mathrm{mL})$ & $0.0033(45)$ & $0.0062(62)$ & $0.510(0.306,0.851)$ \\
Unbound fraction at $3 \mathrm{~h}, \%$ & $0.86(29)$ & $0.86(5)$ & $1.05(0.915,1.21)$ \\
Unbound fraction at $24 \mathrm{~h}, \%$ & $1.04(30)$ & $1.11(9)$ & $0.957(0.801,1.14)$
\end{tabular}

$A U C(0-\infty)$ area under the plasma concentration-time curve from time zero to infinity, $C I$ confidence interval, $C L / F$ apparent oral clearance, $C m a x$ maximum observed plasma concentration, $G L S$ geometric least squares, $N A$ not applicable, $t 1 / 2$ half-life, tmax time to Cmax, $V z / F$ apparent volume of distribution

${ }^{\text {a }}$ Data are geometric mean (coefficient of variation, \%), except for tmax which is denoted as median (range) 
Table 3 Comparison of DTG-glucuronide pharmacokinetic parameters and evaluation of DTG-glucuronide/DTG parameter ratios

\begin{tabular}{|c|c|c|c|}
\hline DTG-glucuronide parameter & Renally impaired (dolutegravir $50 \mathrm{mg}, n=8$ ) & Healthy (dolutegravir $50 \mathrm{mg}, n=8)^{\mathrm{a}}$ & $\begin{array}{l}\text { Renally impaired vs. healthy, } \\
\text { GLS mean ratio }(90 \% \mathrm{CI})\end{array}$ \\
\hline $\operatorname{AUC}(0-\infty), \mu \mathrm{g} \cdot \mathrm{h} / \mathrm{mL}$ & $2.48(78)$ & $0.54(98)$ & $4.30(2.11,8.76)$ \\
\hline $\operatorname{AUC}(0-\mathrm{t}), \mu \mathrm{g} \cdot \mathrm{h} / \mathrm{mL}$ & $2.40(79)$ & $0.49(107)$ & $4.53(2.16,9.49)$ \\
\hline $\mathrm{Cmax}, \mu \mathrm{g} / \mathrm{mL}$ & $0.12(68)$ & $0.04(83)$ & $3.07(1.60,5.89)$ \\
\hline $\mathrm{t} 1 / 2, \mathrm{~h}$ & $12.9(30)$ & $13.0(31)$ & $0.989(0.724,1.35)$ \\
\hline tmax, h & $3.00(1.0,8.0)$ & $1.52(1.0,6.0)$ & NA \\
\hline $\begin{array}{l}\text { DTG-Gluc/DTG } \\
\text { parameter ratio }\end{array}$ & Renally impaired (dolutegravir $50 \mathrm{mg}, n=8)^{\mathrm{b}}$ & Healthy (dolutegravir $50 \mathrm{mg}, n=8)^{\mathrm{b}}$ & $\begin{array}{l}\text { Renally impaired vs. healthy, } \\
\text { estimate of difference }(90 \% \mathrm{CI})\end{array}$ \\
\hline $\operatorname{AUC}(0-\mathrm{t})$ ratio & $0.053(0.041,0.385)$ & $0.011(0.004,0.020)$ & $0.040(0.030,0.140)$ \\
\hline Cmax ratio & $0.045(0.033,0.203)$ & $0.015(0.007,0.023)$ & $0.030(0.020,0.070)$ \\
\hline $\mathrm{t} 1 / 2$ ratio & $1.02(0.930,1.132)$ & $0.836(0.656,1.144)$ & $0.185(-0.020,0.320)$ \\
\hline
\end{tabular}

${ }^{a}$ Data denote geometric mean (CV\%), except for tmax which is denoted as median (range)

${ }^{\mathrm{b}}$ Data for DTG-Gluc/DTG parameter molar ratios are denoted as median (range). Hodges-Lehmann estimates for difference in ratios and $90 \%$ confidence interval are provided

healthy subjects. However, there was considerable overlap in DTG PK parameters between the groups. Median tmax values were similar between the two subject populations, as were the values for fraction unbound in plasma at 3 and $24 \mathrm{~h}$ post-dose. Mean half-life was modestly shorter in the renally impaired $(12.7 \mathrm{~h})$ group compared to the healthy controls $(15.4 \mathrm{~h})$. There was no correlation between creatinine clearance and DTG exposure. It has been well described that renal impairment can lead to a number of changes that may affect the pharmacokinetics of non-renally cleared drugs, including decreased hepatic and intestinal CYP activity, decreased intestinal Pgp and MRP2 activity, and decreased or minimal impact on hepatic OATP activity [12-15]. However, these previously described effects on drug-metabolizing enzymes and transporters would result in a higher exposure in the renal impairment group, in contrast to the overall lower exposure seen in this study.

Previous studies have demonstrated reduced exposure of acidic drugs such as phenytoin in subjects with severe renal impairment with an accompanied higher free fraction in plas$\mathrm{ma}$ and no significant change in the unbound exposure [16]. A possible explanation for the findings in the current study is a reduction in absorption. Patients with severe renal impairment may have alterations in gastrointestinal transit time or bacterial overgrowth in the gastrointestinal tract that may affect drug absorption $[17,18]$. There is evidence that DTG undergoes enterohepatic recirculation [8] and gastrointestinal changes in these subjects may impair this mechanism and contribute to lower exposure in some patients.

The calculated free fraction was similar between renally impaired and normal subjects, which may be explained by the similar albumin levels between the two groups with no change in the association constant. Therefore, the reduction in unbound DTG plasma concentration is driven by the reduction in total DTG plasma concentration, not free fraction. The free fraction (fu) observed in the healthy matched subjects in this study is higher than that observed in a previous study in healthy subjects [19]. The reason for this difference is unclear, but a potential contributor is the slightly lower albumin concentration noted for the healthy subjects in this study resulting in higher free fractions.

Although DTG-Gluc concentrations are much lower than the total DTG concentrations, the plasma exposure of DTGGluc in renally impaired subjects was 3 times (for Cmax) and 4 times (for AUC) higher than in normal subjects. However, the half-life of DTG glucuronide was similar between the two groups and similar to the DTG half-life, indicating formation rate-limited kinetics for the glucuronide metabolite was not altered by severe renal impairment. This suggests that the glucuronide would not be expected to accumulate differentially in renally impaired subjects compared to those with normal function on repeat dosing. From a clinical standpoint, the DTG-Gluc metabolite has no antiviral activity and would not contribute to efficacy in the HIV-infected population. Glucuronidation of DTG disrupts the key metal binding motif of the compound and completely abrogates any antiviral activity resulting from the active site binding to the integrase enzyme. The increased DTG-Gluc concentrations in the renally-impaired population would also not be expected to be a safety concern as the glucuronide is still $<10 \%$ of the exposure of parent DTG based on molar ratios. As such, the glucuronide would not be considered for safety assessment studies according to regulatory guidance on safety testing for metabolites [20]. In addition, the glucuronide is a Phase II metabolite; these metabolites are considered generally more water soluble and less pharmacologically active, and do not require testing [20].

The moderate effect of severe renal impairment in reducing DTG Cmax and AUC by approximately $23-40 \%$ is not considered clinically significant. The reduction is less than 
the $75 \%$ reduction in DTG exposure between the $50 \mathrm{mg}$ and $10 \mathrm{mg}$ once-daily doses observed in a dose-ranging study of DTG in treatment-naïve subjects (SPRING-1) [21]. This trial demonstrated that all DTG doses $(10,25$, and $50 \mathrm{mg})$ combined with a 2 drug nucleoside reverse transcriptase inhibitor backbone were equally efficacious and showed comparable safety. This suggests that the decrease in DTG exposure observed in the current study (approximately $40 \%$ ) would not be clinically relevant in contemporary HIV treatment regimens. Further support for this conclusion is provided by results from co-administration of DTG with darunavir/ritonavir (DRV/r). In a Phase 1 study, DRV/r reduced DTG trough concentration by $38 \%$ [22]. However, in the Phase 3 SAILING study of treatment-experienced but INI-naive subjects, those subjects taking DTG and DRV/r (accounting for $\sim 40 \%$ subjects enrolled) showed equivalent virologic response to the remainder of the DTG-treated population, despite lower exposure [3]. Therefore, it is unlikely that the decrease in exposure seen in this study of subjects with severe renal impairment would have a deleterious effect on virologic response.

This study indicates that no dose adjustment is needed in INInaïve subjects with severe renal impairment (CLcr of $<30 \mathrm{~mL}$ / min, not on renal replacement therapy) and therefore also extends to those with mild and moderate impairment. However, caution should be given to subjects with severe renal impairment and who require higher exposures, such as those with resistance to raltegravir. Furthermore, since metabolic inducers such as efavirenz and tipranavir/ritonavir can decrease DTG concentrations, alternative antiretroviral regimens without such drugs should be considered in subjects with severe renal impairment receiving DTG. Dose recommendations for DTG in end-stage renal disease patients receiving any form of renal replacement therapy cannot be given at the current time, although it is unlikely that such therapy would have a significant effect on DTG PK given the very high protein binding of $>99 \%$.

Financial disclosure Funding for this study was provided by ViiV Healthcare LLC. Stephen Weller, Julie Borland, Shuguang Chen, Mark Johnson, Paul M. Savina, Brian Wynne, Amanda F. Peppercorn, and Stephen C. Piscitelli are employees of GlaxoSmithKline. Toshihiro Wajima is an employee of Shionogi \& Co., LTD.

Open Access This article is distributed under the terms of the Creative Commons Attribution License which permits any use, distribution, and reproduction in any medium, provided the original author(s) and the source are credited.

\section{References}

1. Raffi F, Rachlis A, Stellbrink HJ, Hardy WD, Torti C, Orkin C, Bloch M, Podzamczer D, Pokrovsky V, Pulido F, Almond S, Margolis D, Brennan C, Min S (2013) Once-daily dolutegravir versus raltegravir in antiretroviral-naive adults with HIV-1 infection: 48 week results from the randomised, double-blind, non-inferiority SPRING-2 study. Lancet 381:735-743. doi:10.1016/S0140-6736(12)61853-4
2. G Nichols, A Mills, R Grossberg, et al. (2012) Antiviral activity of dolutegravir in subjects with failure on an integrase inhibitor-based regimen: week 24 phase 3 results from VIKING-3. 11th International Congress on Drug Therapy in HIV Infection (HIV11). Glasgow, November 11-15, 2012. Abstract O232.

3. Cahn P, Posniak AL, Mingrone H, et al (2013) Dolutegravir versus raltegravir in antiretroviral-experienced, integrase-inhibitor-naive adults with HIV: week 48 results from the randomised, double-blind, non-inferiority SAILING study. Lancet 382:700-708

4. Underwood MR, Johns BA, Sato A, Martin JN, Deeks SG, Fujiwara $\mathrm{T}$ (2012) The activity of the integrase inhibitor dolutegravir against HIV-1 variants isolated from raltegravir-treated adults. J Acquir Immune Defic Syndr 61(3):297-301

5. Min S, Song I, Borland J et al (2010) Pharmacokinetics and safety of S/GSK1349572, a next-generation HIV integrase inhibitor, in healthy volunteers. Antimicrob Agents Chemother 54:254-258

6. Min S, Sloan L, DeJesus E, Hawkins T, McCurdy L, Song I, Stroder R, Chen S, Underwood M, Fujiwara T, Piscitelli S, Lalezari J (2011) Antiviral activity, safety, and pharmacokinetics/pharmacodynamics of dolutegravir as 10-day monotherapy in HIV-1-infected adults. AIDS 25(14):1737-45

7. Reese MJ, Savina PM, Generaux GT, Tracey H, Humphreys JE, Kanaoka E, Webster LO, Harmon KA, Clarke JD, Polli JW (2013) In vitro investigations into the roles of drug transporters and metabolizing enzymes in the disposition and drug interactions of dolutegravir, a HIV integrase inhibitor. Drug Metab Dispos 41:35361

8. Castellino S, Moss L, Wagner D, Borland J, Song I, Chen S, Lou Y, Min S, Goljer I, Culp A, Piscitelli SC, Savina PM (2013) Metabolism, excretion, and mass balance of the HIV-1 integrase inhibitor, dolutegravir, in humans. Antimicrob Agents Chemother. doi:10. 1128/AAC.00292-13, ePub ahead of print; 13 May 2013

9. Dreisbach AW, Lertora JJL (2003) The effect of chronic renal failure on hepatic drug metabolism and drug disposition. Semin Dial 16(1): $45-50$

10. Food and Drug Administration. (2010) Guidance for industry: Pharmacokinetics in patients with impaired renal function - study design, data analysis, and impact on dosing and labeling. http://www.fda.gov/ downloads/Drugs/GuidanceComplianceRegulatoryInformation/ Guidances/UCM204959.pdf. Accessed 27 May 2013.

11. TIVICAY (dolutegravir) prescribing information. ViiV Healthcare, Research Triangle Park, NC, August 2013.

12. Pichette V, Leblond F (2003) Drug metabolism in chronic renal failure. Current Drug Metabolism 4:91-103

13. Sun H, Frassetto L, Benet L (2006) Effects of renal failure on drug transport and metabolism. Pharmacology \& Therapeutics 109:1-11

14. Naud J, Michaud J, Boisvert C, Desbiens K, Leblond F, Mitchell A, Jones C, Bonnardeaux A, Pichette V (2007) Down-regulation of intestinal drug transporters in chronic renal failure in rats. $\mathrm{J}$ Pharmacol Exp Ther 320(3):978-985

15. Naud J, Michaud J, Leblond F, Lefrancois S, Bonnardeaux A, Pichette V (2008) Effects of chronic renal failure on liver drug transporters. Drug Met Dispos 36:124-128

16. Liponi DF, Winter ME, Tozer TN (1984) Renal function and therapeutic concentrations of phenytoin. Neurology 34(3):395-7

17. Lefebvre HP, Ferré J-P, Watson DJ A, Brown CA, Serthelon J-P, Laroute V, Concordet D, Toutain P-L (2001) Small bowel motility and colonic transit are altered in dogs with moderate renal failure. Am J Physiol Regul Integr Comp Physiol 281:R230 $\mathrm{R} 238$

18. Strida H, Simrén M, Stotzer P-O, Ringström G, Abrahamsson H, Björnsson ES (2003) Patients with chronic renal failure have abnormal small intestinal motility and a high prevalence of small intestinal bacterial overgrowth. Digestion 67:129-137

19. Song I, Borland J, Savina P, Chen S, Patel P, Wajima T, Peppercorn A, Piscitelli S. (2012) Pharmacokinetics of dolutegravir in subjects with 
moderate hepatic impairment. 19th Conference on Retroviruses and Opportunistic Infections. March 5-8, 2012, Seattle, WA, Abstract 608.

20. Food and Drug Administration. (2008) Guidance for industry: Safety testing of drug metabolites. February 2008. http://www.fda.gov/ downloads/Drugs/GuidanceComplianceRegulatoryInformation/ Guidances/UCM079266.pdf Accessed 27 May 2013.

21. van Lunzen J, Maggiolo F, Arribas JR et al (2012) Once daily dolutegravir (S/GSK1349572) in combination therapy in antiretroviral-naive adults with HIV: planned interim 48 week results from SPRING-1, a dose-ranging, randomised, phase $2 b$ trial. Lancet Infect Dis 12:111-118

22. Song I, Min SS, Borland J, Lou Y, Chen S, Patel P, Ishibashi T, Piscitelli SC (2011) The effect of lopinavir/ritonavir and darunavir/ritonavir on the HIV integrase inhibitor $\mathrm{S} /$ GSK1349572 in healthy participants. J Clin Pharmacol 51(2):237-42 